\title{
Bacterial inclusion bodies: an emerging platform for drug delivery and cell therapy
}

\section{"Unexpectedly, inclusion bodies have turned out to be excellent protein-delivery systems."}

\section{KEYWORDS: amyloids « drug delivery $\approx$ inclusion bodies $\approx$ nanostructured materials} - therapy

\section{Recombinant protein production}

Biological synthesis offers generic advantages over chemical fabrication with regards to versatility, cost-effectiveness and physiological tunability of the production processes. In addition, biological products are essentially biocompatible and do not pose generic toxicity concerns when intended for use as drugs. A representative example of how biosynthesis has impacted biopharmaceuticals is the development of recombinant protein production platforms. This involves the genetic engineering and controlled cultivation of a diversity of cell types (mainly bacteria but also yeast, mammalian cells, insect cells, fungi, microalgae and others) as factories for the production of foreign protein species with therapeutic value. A variety of recombinant hormones, cytokines, growth factors and enzymes have been approved and marketed for protein replacement therapies. Although the accumulated number of recombinant proteins approved by the medicament agencies grows at a rate slower than predicted in early DNA recombinant times, the potential of recombinant production of natural or largely modified proteins is still far from being fully exploited.

\section{Inclusion bodies: bottlenecks in protein production}

A main obstacle to the marketing of recombinant protein drugs is the occurrence of different stresses in the producing cells that results in proteolytic instability of recombinant polypeptides and/or aggregation [1], reducing the yield of the soluble protein or limiting its stability at midstream or even at postproduction stages [2]. In bacteria, the most explored cell factory for protein production, recombinant polypeptides often aggregate (partially or totally) as refractile and insoluble clusters termed inclusion bodies (IBs). This is a consequence of the nonphysiologically high amounts of foreign polypeptides accumulating in the factory cell, which tend to cross-interact. As a result of this, IBs are formed as spherical or slightly ovoid proteinaceous particles ranging between 50 and $700 \mathrm{~nm}$ in size that are highly enriched with the recombinant protein and also contain minor amounts of cell proteins (mainly chaperones), traces of nucleic acids and other cell components. Microbiologists have explored a spectrum of approaches to minimize IB formation and consequently increase the yield of the soluble protein. Reducing the bacterial culture temperature, fusing peptidic tags to favor protein solubility or coexpressing chaperone genes along with the target gene show irregular effects that are highly product-dependent and not suitable for general application. Upon cell wall disruption, IBs remain stable and sediment with the insoluble cell fraction. These particles can then be easily purified by repeated centrifugation and washing steps, upon enzymatic cell lysis or mechanical cell disruption. Because of the relative purity of the recombinant protein within IBs, the mechanical stability of these particles, their easy separation from cell debris and the cost-effective IB isolation processes, isolated IBs may be used as a convenient source of recombinant protein that can be recovered in vitro, in soluble form, upon the application of protein denaturation and refolding treatments.

Despite the belief that IBs were composed of misfolded and nonfunctional versions of the target protein [3], recent research on the physiology of IB formation has revealed, as a general trait, that at least a significant fraction of IB polypeptides are biologically functional and therefore properly folded [4]. This observation has conduced to the development of protocols for in vitro protein recovery from IBs in which functional IB proteins are released without the

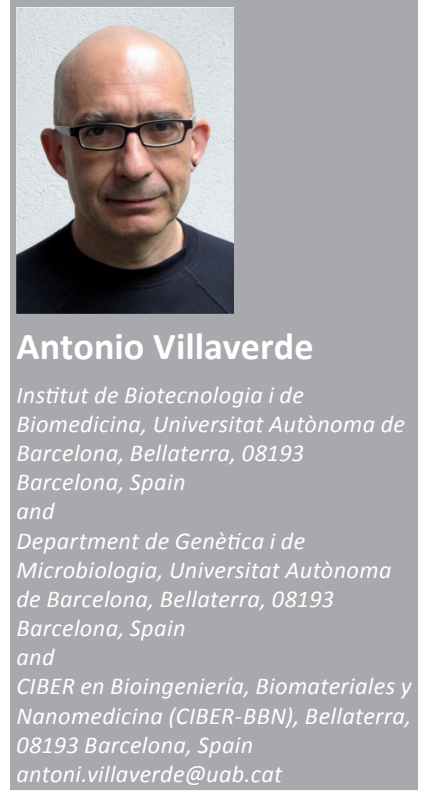

Future
Medicine
part of 
application of denaturing treatments [5,6]. In biotechnology, functional IBs formed by enzymes can be exploited as immobilized catalysts with high operational stability [7]. The potential of IBs as biocatalyzers is based not only on the functionality of IB proteins but also on the mechanical stability and porosity of the particles that allows proper mass transfer.

\section{IBs as amyloid bionanomaterials}

The interest in bacterial IBs has also been revitalized by the emergence of prion research, by the progressive understanding of conformational diseases at a molecular level and, especially, by the need for fast, simple and inexpensive in vivo models of protein aggregation [8]. Determining the physiology of IB formation in living bacteria and the incorporation of techniques for the structural analysis of proteins (e.g., Fourier transform infrared spectroscopy and circular dichroism) in IB research has allowed us to gain insights into the biology and molecular organization of bacterial IBs. Under structural examination and according to amyloid dye staining, IB proteins are organized in an amyloid-like architecture [9] in which at least a significant fraction of the polypeptides occur as fibers [10]. Furthermore, the volumetric growth of IBs in biosynthetically active bacteria is a sequence-specific seeding event, which accounts for the high protein purity of the recombinant protein in IBs [11]. The cross-molecular sterospecific protein-protein contacts that determine sequence-specific aggregation require secondary structure of the partner polypeptide chains [12]. Therefore, IB proteins are enriched in native or native-like structures resulting in biologically active IBs [9], from which functional polypeptides can be released, even by nondenaturing methods [6,5]. How tightly cross$\beta$-pleated sheet interactions (rending amyloid fibers) and sterospecific contacts between homologous protein patches (rending clusters of functional proteins) might coexist in single particles remains an unsolved question. Irrespective of the precise molecular arrangements of IB polypeptides, their mechanical stability make these particles appealing bionanomaterials [13], as has also been proposed for natural amyloids [14]. Indeed, we have successfully explored IBs formed by proteins lacking any relevant biological activity as nanoparticles for the bottom-up modification of 2D surfaces, to favor their colonization by mammalian cells [15]. The mechanical properties of IBs at the nanoscale seem to be in the range sensed by mammalian cells, stimulating cell proliferation through mechanical stimuli.

\section{IBs as nanopills for drug delivery}

Interestingly, mammalian cells in contact with purified IBs do not show any sign of toxicity. This fact, combined with the biological activity of IB proteins, prompted us to explore the potential healing effects of IBs formed by therapeutic proteins, when added to the culture medium as 'nanopills' for protein replacement. A few hours after exposure to reporter (fluorescent) IBs, these entities penetrated cells as morphologically stable particles, reaching both cytoplasmic and nuclear compartments [16]. In addition, IBs formed by the therapeutic proteins Hsp70, LIF, catalase and dihydrofolate reductase increased the viability of target cells challenged with different stresses [16]. This was indicative not only of functionality but also of bioavailability of the IB-embedded proteins. How IBs of up to $400 \mathrm{~nm}$ cross the plasma and nuclear membranes while maintaining the cellular integrity is a question that deserves further analysis. Since the morphology of IBs remained essentially unchanged once the biological effect was made apparent, this must be associated to the slow release of functional protein monomers. The nanopill concept, as a potential platform for controlled protein packaging (in bacterial factories) and further release (in target mammalian cells), has been further confirmed by the partial reconstruction of the cell cytoskeleton through exposure to keratin 14 IBs [17].

\section{Do IBs mimic natural amyloidal secretory granules?}

In higher organisms, amyloid deposits have been closely associated with degenerative diseases, but their in vivo self-organization as fibers or regular particles through the ordered cross-interaction of forming polypeptides has prompted some authors to consider them (as well as those originating in nonrecombinant bacteria such as chaplins and curli) as intriguing bionanomaterials $[14,18]$. In this context and in parallel to IB research, the occurrence of eukaryotic amyloids formed by functional proteins is being progressively recognized. In fact, many protein hormones are stored in secretory granules as amyloids fibers, from which functional monomeric building blocks are progressively released during glandular secretion [19]. This reveals an unexpected natural ability of amyloids as reservoirs of functional proteins in healthy organisms, which may have been evolutively adapted for slow release of their components $[19,20]$. Fitting with the observation of bacterial IBs acting as nanopills, protein organization as amyloids in the endocrine system is a 
natural mechanism for the storage of bioactive and releasable proteins.

\section{Conclusion}

In the context of the recent discovery of natural amyloids for the slow release of functional hormones, recombinant bacteria should be reconsidered not only as a conventional producer of soluble polypeptides for biopharmaceuticals, but also as a promising cell factory for the controlled biofabrication of amyloid-based protein nanoparticles (IBs). Unexpectedly, IBs have turned out to be excellent protein-delivery systems. As essentially any desired therapeutic protein can be produced in bacteria as IBs (provided no post-translational modifications absent in prokaryotes are required for the biological activity), bacterial factories would be extremely useful for the production of nanostructured particulate materials with the potential to supply functional proteins (their building blocks) to target biological systems (administered as nanopills). Preliminary observations showing full tolerance to oral delivery of high doses of IBs [16] opens a plethora of exciting opportunities for new pharmacological preparations based on these materials, as a generic technological platform for delivery of protein drugs in innovative therapies.

\section{Financial \& competing interests disclosure \\ The author appreciates the financial support for research on inclusion bodies through grants MINECO BFU2010-17450 and AGAUR 2009SGR-108, from the Biomedical Research Networking Center in Bioengineering, Biomaterials and Nanomedicine (CIBER-BBN) and from ICREA (through an ICREA Academia award). A Villaverde is co-inventor of a patent (WO2010131117A1, licensed to Janus Development SL), covering the use of bacterial inclusion bodies as therapeutic agents. The author has no other rel- evant affiliations or financial involvement with any organi- zation or entity with a financial interest in or financial conflict with the subject matter or materials discussed in the manuscript apart from those disclosed. \\ No writing assistance was utilized in the production of this manuscript.}

\section{References}

1 Gasser B, Saloheimo M, Rinas U et al. Protein folding and conformational stress in microbial cells producing recombinant proteins: a host comparative overview. Microb. Cell Fact. 7, 11 (2008).

2 Vazquez E, Corchero JL, Villaverde A. Post-production protein stability: trouble beyond the cell factory. Microb. Cell Fact. 10(1), 60 (2011).

3 Baneyx F, Mujacic M. Recombinant protein folding and misfolding in Escherichia coli. Nat. Biotechnol. 22(11), 1399-1408 (2004).

4 Gonzalez-Montalban N, Garcia-Fruitos E, Villaverde A. Recombinant protein solubility-does more mean better? Nat. Biotechnol. 25(7), 718-720 (2007).

5 Peternel S, Grdadolnik J, Gaberc-Porekar V, Komel R. Engineering inclusion bodies for non denaturing extraction of functional proteins. Microb. Cell Fact. 7(1), 34 (2008).

6 Upadhyay AK, Murmu A, Singh A, Panda AK. Kinetics of inclusion body formation and its correlation with the characteristics of protein aggregates in Escherichia coli. PLoS ONE 7(3), e33951 (2012).

7 Garcia-Fruitos E, Vazquez E, Díez-Gil C et al. Bacterial inclusion bodies: making gold from waste. Trends Biotechnol. 30(2), 65-70 (2012).

8 Garcia-Fruitos E, Sabate R, de Groot NS, Villaverde A, Ventura S. Biological role of bacterial inclusion bodies: a model for amyloid aggregation. FEBS J. 278(14), 2419-2427 (2011).

9 Ventura S, Villaverde A. Protein quality in bacterial inclusion bodies. Trends Biotechnol. 24(4), 179-185 (2006).

10 Morell M, Bravo R, Espargaro A et al. Inclusion bodies: specificity in their aggregation process and amyloid-like structure. Biochim. Biophys. Acta 1783(10), 1815-1825 (2008).

11 Carrio M, Gonzalez-Montalban N, Vera A, Villaverde A, Ventura S. Amyloid-like properties of bacterial inclusion bodies. J. Mol. Biol. 347(5), 1025-1037 (2005).

12 Speed MA, Wang DI, King J. Specific aggregation of partially folded polypeptide chains: the molecular basis of inclusion body composition. Nat. Biotechnol. 14(10), 1283-1287 (1996).

13 Villaverde A. Nanotechnology, bionanotechnology and microbial cell factories. Microb. Cell Fact. 9, 53 (2010).

14 Cherny I, Gazit E. Amyloids: not only pathological agents but also ordered nanomaterials. Angew. Chem. Int. Ed. Engl. 47(22), 4062-4069 (2008).

15 Seras-Franzoso J, Díez-Gil C, Vazquez E et al. Bioadhesiveness and efficient mechanotransduction stimuli synergistically provided by bacterial inclusion bodies as scaffolds for tissue engineering. Nanomedicine (Lond.) 7(1), 79-93 (2012).

16 Vazquez E, Corchero JL, Burgueno JF et al. Functional inclusion bodies produced in bacteria as naturally occurring nanopills for advanced cell therapies. Adv. Mater. 24(13), 1742-1747 (2012).

17 Liovic M, Ozir M, Bedina ZA, Peternel S, Komel R, Zupancic T. Inclusion bodies as potential vehicles for recombinant protein delivery into epithelial cells. Microb. Cell Fact. 11(1), 67 (2012).

18 Iconomidou VA, Hamodrakas SJ. Natural protective amyloids. Curr. Protein Pept. Sci. 9(3), 291-309 (2008).

19 Maji SK, Perrin MH, Sawaya MR et al. Functional amyloids as natural storage of peptide hormones in pituitary secretory granules. Science 325(5938), 328-332 (2009).

20 Maji SK, Schubert D, Rivier C, Lee S, Rivier JE, Riek R. Amyloid as a depot for the formulation of long-acting drugs. PLoS Biol. 6(2), e17 (2008). 